vereinbarung - auch Honorar-, Konsiliar- oder Belegärzte $\mathrm{zu}$ verstehen sein, ist demgegenüber fernliegend (ähnlich LG Hamburg, Urt. v. 16.10.2015 - 332 O 214/14 -, juris, Rdnr. 14). Soweit das Berufungsgericht seine abweichende Auffassung auf das Urt. des LG Stuttgart v. 4.5.2016 (13 S 123/15, MedR 2017, 322) stützen will, wird übersehen, dass die dortige Vereinbarung über wahlärztliche Leistungen die Wahlarztkette pauschal auf alle an der Behandlung beteiligten Ärzte erstreckte. Demgegenüber setzt die hier streitige Klausel gerade einschränkend voraus, dass die wahlärztlichen Leistungen von liquidationsberechtigen „Ärzten des Krankenhauses“ erbracht werden.

[27] c) Diese Auslegung wird ferner durch den Gesamtzusammenhang der Regelungen in der Wahlleistungsvereinbarung und den darin in Bezug genommenen Unterlagen bestätigt.

[28] In den ,Weiteren Vertragsbedingungen“ auf der Rückseite der Wahlleistungsvereinbarung wird unter ausdrücklichem Hinweis auf $\int 17$ KHEntgG klargestellt, dass bei der Inanspruchnahme wahlärztlicher Leistungen die Wahl nicht auf einzelne „Ärzte des Krankenhauses“ beschränkt werden kann. Vor allem aber wird in den Vertragsbedingungen im Zusammenhang mit der Verhinderung des Wahlarztes der jeweiligen Fachabteilung auf die „Liste der Leitenden Ärzte und deren Vertreter" (Wahlarztliste) Bezug genommen, deren Erhalt der Patient gemäß Nr. 4 der auf der Vorderseite der Wahlleistungsvereinbarung abgedruckten „Hinweise und Vereinbarungen“ durch seine Unterschrift ausdrücklich bestätigt. Aus der dem Patienten ausgehändigten „Anlage zur Wahlleistungsvereinbarung ärztliche Leistungen“ (das ist die vorgenannte Wahlarztliste) ergibt sich, dass die wahlärztlichen Leistungen ausschließlich von den leitenden Ärzten der Fachabteilungen (Chefärzte) beziehungsweise von den angegebenen Chefarztstellvertretern (als besonders benannte Wahlärzte) erbracht werden. Als ständige Vertreter des Chefarztes beziehungsweise des besonders benannten Wahlarztes werden ausschließlich Oberärzte des Krankenhauses aufgeführt, bei denen es sich nicht um Honorar-, Beleg- oder Konsiliarärzte handelt. Damit liegt auch aus der Sicht eines durchschnittlichen, rechtlich nicht vorgebildeten Patienten klar auf der Hand, dass in die Wahlarztkette nur solche Ärzte eingebunden sind, die in einem festen Anstellungs- oder Beamtenverhältnis zu dem Krankenhausträger stehen. Entgegen der Auffassung des Berufungsgerichts kann dahinstehen, ob die Wahlarztliste Vertragsbestandteil geworden ist, wogegen im Übrigen nach Auffassung des Senats keine Bedenken bestehen. Ihre Aushändigung im Zusammenhang mit dem Abschluss der Wahlleistungsvereinbarung dient der Information des Patienten. Sie stellt daher jedenfalls ein zulässiges Auslegungsmittel dar (vgl. BAG, NJW 2011, 101, Rdnr. 51).

[29] c) Dafür, dass die Vertragsparteien den Inhalt der Wahlleistungsvereinbarung übereinstimmend abweichend vom objektiven Sinngehalt der Klausel verstanden haben, ist nichts ersichtlich. [...]

$[30][\ldots]$

https://doi.org/10.1007/s00350-018-5073-y

\section{Anmerkung zu BGH Urt. v. 19.4.2018 - III ZR 255/17 (LG Heidelberg)}

\section{Markus Wessel}

Die Entscheidung des BGH zum Honorararzt ${ }^{1}$ führte $\mathrm{zu}$ Missverständnissen ${ }^{2}$, obwohl das $\mathrm{BVerfG}^{3}$ eine Verfassungsbeschwerde ablehnte, weil der Entscheidung des BGH

Dr. iur. Markus Wessel, Rechtsanwalt,

Schlüterstraße 39 in 10629 Berlin, Deutschland zum Honorararzt keine Bedeutung für Ärzte des Krankenhauses zukommt ${ }^{4}$. Das LG Stuttgart ${ }^{5}$ entschied, Wahlleistungsvereinbarungen müssten den Wortlaut des $\$ 17$ Abs. 3 S. 1 KHEntgG ,angestellte oder beamtete Ärzte“ zitieren. Die AG Hamburg-St. Georg ${ }^{6}$ und Ahrensburg ${ }^{7}$ verlangten ein wörtliches Zitat, nachdem das LG Hamburg bereits $2015^{8}$ und $2018^{9}$ feststellte, dass $\$ 17$ Abs. (3) S. 1 KHEntgG nicht im Sinne eines Zitiergebots zu verstehen sei, weil die Formulierung „Ärzte des Krankenhauses“ eine geringfügige Abweichung vom Wortlaut ohne inhaltliche Bedeutung ist. Die BGH-Entscheidung vom 19.4.2018 bestätigt das Ergebnis des LG Hamburg.

1) BGH, Urt. v. 16.10.2014 - III ZR 85/14 - (sog. Honorararztentscheidung), juris; BGH, Urt. v. 14.1.2016 - III ZR 107/15 - (betr. Zustandekommen der Wahlleistungsvereinbarung), juris.

2) So bereits Dahm, MedR 2017, 324-325, wenn ein Krankenhaus keine beamteten Ärzte habe.

3) BVerfG, Beschl. v. 3.3.2015 - 1 BvR 3226/14.

4) BVerfG, a.a. O., Rdnrn. $21 \mathrm{ff}$.

5) LG Stuttgart, MedR 2017, 322

6) AG Hamburg-St. Georg, Urt. v. 1.8.2017 - 919 C 58/17 -; Urt. v. 11.7.2017-921 C 214/16

7) AG Ahrensburg, Urt. v. 6.3.2018 - 47 C 215/16 -; Urt. v. 2.2.201744 C $189 / 16$-; Urt. v. 2.2.2017 - 44 C 219/16.

8) LG Hamburg, Urt. v. 16.10.2015 - 332 O 214/14 -, juris.

9) LG Hamburg, Beschl. v. 15.12.2018 - 322 O 85/17 -; Beschl. v. 11.1.2018- 332 O 118/17.

\section{Verletzung des Anspruchs auf rechtliches Gehör durch Übergehen von Klägervortrag zu einem möglichen groben Behandlungsfehler}

GG Art. 103 Abs. 1; ZPO §544 Abs. 7

Das Gericht verletzt den Anspruch der Partei auf rechtliches Gehör aus Art. 103 Abs. 1 GG, wenn es bei seiner Annahme, ein Behandlungsfehler sei nicht als grober Fehler anzusehen, von der Partei vorgetragene, für die Bewertung des Behandlungsgeschehens erhebliche Umstände übergeht (hier: Vortrag dahin, der Fehler beruhe auf einem Organisations- bzw. Übertragungsfehler, nicht auf einer Abwägung der Chancen und Risiken der unterbliebenen Befundung).

BGH, Beschl.v. 7.11.2017 - VI ZR 173/17 (OLG Frankfurt/Main)

Problemstellung: Die Gehörsrüge gewinnt im (Zahn-)Arzthaftungsrecht immer wieder an Bedeutung (vgl. z. B. BVerfG, Beschl. v. 17.4.2012 - 1 BvR 3071/10 -, GesR 2012, 418, dazu Finn, GuP 2012, 153 ff.). Im Rahmen einer Nichtzulassungsbeschwerde hat der VI. Zivilsenat des BGH nun mit seinem Beschl. vom 7.11.2017 wegen Gehörsverletzung das Berufungsurt. aufgehoben und die Streitsache zur neuen Verhandlung und Entscheidung an das OLG zurückverwiesen ( 5544 Abs. 7 ZPO). Inhaltlich ging es um eine Beweislastentscheidung des Berufungsgerichts, die möglicherweise anders ausgefallen wäre, wenn der klägerische Sachvortrag zum Grund der unterbliebenen Befunderhebung - Organisationsdefizit im Krankenhaus statt (bewusster) ärztlicher Abwägungsentscheidung - nicht übergangen worden wäre.

Eingesandt vom BGH-Entscheidungsversand, Karlsruhe; bearbeitet von Professor Dr. iur. Markus Finn,

Lehrbeauftragter der Charité sowie der MHB Theodor Fontane, Professur für Recht im Gesundheitswesen,

Alfons-Goppel-Platz 1, 95028 Hof, Deutschland 